\title{
Free polar motion of a triaxial and elastic body in Hamiltonian formalism: Application to the Earth and Mars
}

\author{
M. Folgueira ${ }^{1,2}$ and J. Souchay ${ }^{2}$ \\ ${ }^{1}$ Instituto de Astronomía y Geodesia (UCM - CSIC), Facultad de Ciencias Matemáticas, Universidad Complutense de Madrid, \\ Ciudad Universitaria, 28040 Madrid, Spain \\ e-mail: martafl@mat.ucm.es \\ 2 Observatoire de Paris, SYRTE, UMR 8630 du CNRS, 61 avenue de l'Observatoire, 75014 Paris, France \\ e-mail: Jean.Souchay@obspm.fr
}

Received 18 May 2004 / Accepted 25 October 2004

\begin{abstract}
The purpose of this paper is to show how to solve in Hamiltonian formalism the equations of the polar motion of any arbitrarily shaped elastic celestial body, i.e. the motion of its rotation axis (or angular momentum) with respect to its figure axis. With this aim, we deduce from canonical equations related to the rotational Hamiltonian of the body, the analytical solution for its free polar motion which depends both on the elasticity and on its moments of inertia. In particular, we study the influence of the phase angle $\delta$, responsible for the dissipation, on the damping of the polar motion. In order to validate our analytical equations, we show that, to first order, they are in complete agreement with those obtained from the classical Liouville equations.

Then we adapt our calculations to the real data obtained from the polar motion of the Earth (polhody). For that purpose, we characterize precisely the differences in radius $J-\chi$ and in angle $l-\theta$ between the polar coordinates $(\chi, \theta)$ and $(J, l)$ representing respectively the motion of the axis of rotation of the Earth and the motion of its angular momentum axis, with respect to an Earth-fixed reference frame, after showing the influence of the choice of the origin on these coordinates, and on the determination of the Chandler period as well. Then we show that the phase lag $\delta$ responsible for the damping for the selected time interval, between Feb. 1982 and Apr. 1990, might be of the order of $\delta \approx 6^{\circ}$, according to a numerical integration starting from our analytical equations. Moreover, we emphasize the presence in our calculations for both $\chi$ and $\theta$, of an oscillation with a period $T_{\text {Chandler }} / 2$, due to the triaxial shape of our planet, and generally not taken into account.

In a last step, we apply our analytical formulation to the polar motion of Mars, thus showing the high dependence of its damping on the poorly known value of its Love number $k$. Moreover we emphasize the large oscillations of Mars' polar motion due to the triaxiality of this planet.
\end{abstract}

Key words. methods: analytical - solar system: general

\section{Introduction}

The torque-free rotational motion of a given body, sometimes called the Euler-Poinsot problem, corresponds to the case for which no torque is exerted by any external body. The application of such motion to a rigid Earth is a classical problem already discussed by several authors. Usually it has been treated from the analytical point of view by solving either Euler's equations of motion or the Hamiltonian equations of the torque-free motion for an axially symmetric Earth - see for instance Kinoshita (1972a,b, 1992). In a previous paper (Souchay et al. 2002), we have applied a numerical integration method based on the Runge-Kutta-Fehlberg algorithm, to compare the rotation of several typical celestial bodies with different shapes, such as the Earth, Mars and Eros, considered as rigid bodies.

In this paper we consider an arbitrary elastic and triaxial body, and we analyze the analytical solution of its torque-free rotational motion, taking into particular account the elastic deformation caused by the centrifugal force due to rotation. We first show the process for the formulation of such equations of motion expressed in terms of Andoyer's variables and its canonically conjugate variables.

The Hamiltonian formulation for the rotation of an elastic body such as the Earth has been discussed in detail by, to the best of our knowledge, Sevilla \& Romero (1985), Getino \& Ferrándiz (1990, 1991), Kubo (1991) and Stavinschi \& Souchay (1994). In this study, we have followed Kubo's procedure and we have amplified some aspects of his presentation, considering a triaxial body instead of a body with rotational symmetry. Moreover, we have shown the equivalence between our Hamiltonian formulation, and the Liouville equations obtained in a quite different manner.

During the thirteen years that have passed since Kubo (1991), the level of accuracy in the observational techniques, 
such VLBI (Very Long Baseline Interferometry), GPS (Global Positioning System) and LLR (Lunar Laser Ranging), has increased enormously. As an application of the theoretical developments above, we not only show that at the level of 1 mas some variations related to the triaxial shape of the Earth and generally not considered have to be taken into account at the present date. We also evaluate the combined effects on the polar motion of the elasticity, the triaxiality of the Earth and the poorly known lag coefficient $\delta$ which is responsible for damping. For this purpose we choose real data taken from IERS (International Earth Rotation and Reference System Service).

Our treatment consists of a detailed discussion and mathematical modelling for the damping of the free rotational motion with the purpose of explaining better how the Chandler wobble is maintained in the presence of such dissipation. The nature of the continuous excitation for the Earth (such as the response of the oceans, fluidity of the core, atmospheric excitation, electromagnetic processes, etc.) is not discussed here. For more information, the reader is referred to the works of Dehant et al. (1999), Nastula \& Ponte (1999) and McCarthy \& Petit (2003), for instance. Therefore, our goal is a refinement, at the present accuracy, of the mathematical model, to reduce the residual deviation of the model from reality.

The Hamiltonian variational equations seem to be more convenient and useful in many respects, since such equations enable us not only to separate precisely the different terms according to the different aspects considered in this paper (triaxiality, elasticity and time lag), but also to make practical computations for the study of the free polar motion of an arbitrary celestial body. By integrating the general variational equations for the polar coordinates $l$ (angle) and $J$ (amplitude), we show that the corresponding solutions for these two parameters have secular and periodic parts, this latter caused by three different effects taken into account here.

For the case of Mars' free polar motion, we have analyzed the extended analytical solutions coming from the Hamiltonian formalism to show how the phase shift, $\delta$, also plays an important role in its motion. A study of the normal modes of a triaxial planet such as the Earth and Mars using the Liouville method can be seen in Van Hoolst \& Dehant (2002).

\section{Notation and definitions}

For later use, we list in Table 1 the fundamental arguments which allow us to write the equations of the torque-free rotational motion of an elastic body. We also present in the same table other useful quantities, such as constants and geodetic parameters, considered in the present paper. For this purpose, we have chosen the same notation as in Kinoshita (1977), Moritz \& Mueller (1987), Kubo (1991) and Souchay et al. (2002) in order to establish homogeneity of notation. The interested reader is referred to the above mentioned articles and textbook for more details.
Table 1. Notation

\begin{tabular}{|c|c|}
\hline Symbol & Definition \\
\hline$\{O ; X, Y, Z\}$ & Inertial reference frame \\
\hline$\{O ; x, y, z\}$ & Arbitrary body-fixed axes \\
\hline$\left(\omega_{1}, \omega_{2}, \omega_{3}\right)$ & $\begin{array}{l}\text { The components of the angular rotational vector } \\
\vec{\omega}\end{array}$ \\
\hline$(L, G, H)$ & Andoyer's variables \\
\hline$(l, g, h)$ & Canonically conjugate variables to $(L, G, H)$ \\
\hline$(\chi, \theta)$ & $\begin{array}{l}\text { Polar coordinates of the motion of the axis of } \\
\text { rotation of the body with respect to its figure axis }\end{array}$ \\
\hline$J$ & $\begin{array}{l}\text { Angle between angular momentum equator and } \\
\text { the equator of figure }(O ; x, y)\end{array}$ \\
\hline$T$ & Kinetic energy \\
\hline $\mathcal{K}=T$ & Hamiltonian for the free rotation \\
\hline$\vec{M}$ & Angular momentum vector \\
\hline$C=C^{R}+c$ & Inertia tensor \\
\hline$C^{R}$ & $\begin{array}{l}\text { Inertia tensor of an undeformed body whose } \\
\text { principal axes of inertia coincide with the } \\
\text { body-fixed axes }\end{array}$ \\
\hline$c$ & $\begin{array}{l}\text { Tensor which takes into account the deviation of } \\
\text { a deformable body from the rigid model }\end{array}$ \\
\hline$A_{0}, B_{0}, C_{0}$ & Principal moments of inertia of a rigid body \\
\hline$k$ & Love number \\
\hline$k_{\mathrm{s}}$ & Secular Love number \\
\hline$v$ & Intermediary parameter \\
\hline$C_{0}^{\prime}$ & Intermediary parameter \\
\hline$\varepsilon$ & Triaxial parameter \\
\hline$\alpha, \beta, \gamma, \rho, \tau$ & Elastic parameters \\
\hline$\delta$ & Phase lag \\
\hline$\sigma_{\mathrm{E}}$ & Euler frequency \\
\hline$\sigma_{\mathrm{C}}$ & Chandler frequency \\
\hline
\end{tabular}

\section{Equations of the torque-free rotational motion of an elastic body in terms of Andoyer variables: Extension of Kubo's procedure}

\subsection{Expression of the Hamiltonian of the torque-free rotational motion.}

In this section, we will generalize the equations of the torque-free rotational motion of a rigid body in terms of the Andoyer variables as discussed in Souchay et al. (2002), to the case of a deformable body and also consider two kinds of shape-configuration, a quasi axisymmetric body and a body with a triaxial form. For this purpose, we will also summarize here some basic facts and formulas which are needed to describe the equations. For details, we refer the reader to textbooks such as Munk \& MacDonald (1975), Lambeck (1980) and Moritz \& Mueller (1987).

The basic equation

$\mathcal{K}=T=\frac{1}{2} \vec{M}^{T} C^{-1} \vec{M}$ 
expresses the Hamiltonian of the torque-free rotational motion (equal to the kinetic energy, in this case) in terms of the angular momentum $\vec{M}$ and the inertia tensor $C$. It holds for the rotation of an arbitrary body, rigid or elastic, whenever we chose as the body-fixed axes $\{x, y, z\}$, the Tisserand axes (Munk \& MacDonald 1975, p.10). By taking these axes, the relative, or deformational, angular momentum $\vec{h}=\int_{T} \vec{x} \times \vec{u}-$ caused by the existence of a residual deformation velocity $\vec{u}$, see for instance (Moritz \& Mueller 1987, pp. 110-115) - can be made zero and the angular momentum vector $\vec{M}$ takes the same form as for a rigid body. In the following, we will always use Tisserand axes. Notice also that, in the case of a deformable body, the inertia tensor $C$ will be a function of time since the shape of the body will, in general, change with the time. Then, the inertia tensor $C$ can be written as:

$C=C^{R}+c=\left(\begin{array}{lll}A & F & E \\ F & B & D \\ E & D & C\end{array}\right)$

where,

$C^{R}=\left(\begin{array}{ccc}A_{0} & 0 & 0 \\ 0 & B_{0} & 0 \\ 0 & 0 & C_{0}\end{array}\right)$

corresponds to an rigid body whose principal axes of inertia coincide with the body-fixed axes and whose principal moments of inertia $A_{0}, B_{0}$ and $C_{0}$ are constant in time (Souchay et al. 2002), and

$c=\left(\begin{array}{lll}c_{11} & c_{12} & c_{13} \\ c_{12} & c_{22} & c_{23} \\ c_{13} & c_{23} & c_{33}\end{array}\right)$

takes into account the deviation of a deformable body from the rigid model, so that the elements $c_{i j}$ are small quantities. From Eqs. (2) and (4), we have: $A=A_{0}+c_{11}, B=B_{0}+c_{22}, C=$ $C_{0}+c_{33}, D=c_{23}, E=c_{13}$ and $F=c_{12}$.

Taking into account the above considerations, the angular momentum vector $\vec{M}$ is written as (Souchay et al. 2002):

$\vec{M}^{T}=\left(L_{x}, L_{y}, L_{z}\right)=\left(\sqrt{G^{2}-L^{2}} \sin l, \sqrt{G^{2}-L^{2}} \cos l, L\right)$.

We substitute Eqs. (5) and (2) into Eq. (1). The result is, after some developments:

$$
\begin{aligned}
\mathcal{K}= & \frac{1}{2 \Delta}\left\{\left[\left(B C-D^{2}\right) \sin ^{2} l+\left(A C-E^{2}\right) \cos ^{2} l\right]\left(G^{2}-L^{2}\right)\right. \\
& +\left(A B-F^{2}\right) L^{2}-(C F-D E)\left(G^{2}-L^{2}\right) \sin 2 l \\
& \left.-2 L \sqrt{G^{2}-L^{2}}[(B E-F D) \sin l+(A D-E F) \cos l]\right\}
\end{aligned}
$$

$\Delta$ being the determinant of inertia tensor $C$, that is to say,

$\Delta=A B C-A D^{2}-B E-C F^{2}+2 D E F$.

Note: Expression (6) has the same form as that obtained by (Kubo 1991) using a different procedure.
$D, E$ and $F$ are considered small quantities whose products, squares and higher powers can be neglected. Equation (6) then reduces to:

$$
\begin{aligned}
\mathcal{K}= & \frac{1}{2 A B C}\left\{\left[B C \sin ^{2} l+A C \cos ^{2} l\right]\left(G^{2}-L^{2}\right)+A B L^{2}\right. \\
& -C F\left(G^{2}-L^{2}\right) \sin 2 l \\
& \left.-2 L \sqrt{G^{2}-L^{2}}[B E \sin l+A D \cos l]\right\} .
\end{aligned}
$$

Considering rotational symmetry $(A=B)$, Eq. (7) reduces to the simple form:

$$
\begin{aligned}
\mathcal{K}= & \frac{1}{2 A}\left(G^{2}-L^{2}\right)+\frac{1}{2 C} L^{2}-\frac{F}{2 A^{2}}\left(G^{2}-L^{2}\right) \sin 2 l \\
& -\frac{1}{A C} L \sqrt{G^{2}-L^{2}}[E \sin l+D \cos l] .
\end{aligned}
$$

\section{Rotational deformation:}

If the elastic body rotates about an axis which deviates from the axis of symmetry of the body, then centrifugal forces tend to distort it and therefore this distortion causes variations in the tensor of inertia. This effect is known as rotational deformation, which is the only one to take into account in the study of the torque-free rotational motion of an elastic body. Periodic variations in the tensor arising from rotational deformation are given by (Munk \& MacDonald 1975, p. 25):

$c_{11}=c_{22}=-\frac{2}{3} \frac{k}{k_{s}}\left(C_{0}-A_{0}\right) m_{3}$

$c_{33}=\frac{4}{3} \frac{k}{k_{s}}\left(C_{0}-A_{0}\right) m_{3}$

$D=\frac{k}{k_{s}}\left(C_{0}-A_{0}\right) m_{2}$

$E=\frac{k}{k_{s}}\left(C_{0}-A_{0}\right) m_{1}$

$F=0$

where, $k$ is a Love number, $k_{\mathrm{s}}$ is the secular Love number equal to $\frac{3 \kappa^{2}\left(C_{0}-A_{0}\right)}{a^{5} \Omega^{2}}\left(\kappa^{2}\right.$ denotes the gravitational constant and $a$ is the Earth's equatorial radius), $m_{1}$ and $m_{2}$ express deviations of the rotation axis from the $z$-axis and $m_{3}$ express variations of the rotational speed. That is to say, the rotation vector $\vec{\omega}$ is written, in the case of an elastic body, as:

$\vec{\omega}=\vec{\omega}_{0}+\overrightarrow{\delta \omega}$

with

$\vec{\omega}_{0}^{T}=(0,0, \Omega)$,

which corresponds to a rotation with constant angular velocity $\Omega$ around the $z$-axis, and

$\overrightarrow{\delta \omega}^{T}=\left(m_{1}, m_{2}, m_{3}\right) \Omega$.

For Tisserand axes, we can also use the following relations for the components of the angular-momentum vector (Kinoshita 1977):

$\sqrt{G^{2}-L^{2}} \sin l=A \omega_{1}$
$\sqrt{G^{2}-L^{2}} \cos l=B \omega_{2}$

$L=C \omega_{3}$. 
The variations $c_{11}, c_{22}, c_{33}$ and $\overrightarrow{\delta \omega}$ are small quantities whose products and squares are too small to be of interest in the present study. Then the components of $\overrightarrow{\delta \omega}$ can be written, in terms of Andoyer variables, as:

$$
\begin{aligned}
& m_{1}=\frac{1}{A_{0} \Omega} \sqrt{G^{2}-L^{2}} \sin l \\
& m_{2}=\frac{1}{B_{0} \Omega} \sqrt{G^{2}-L^{2}} \cos l \\
& m_{3}=\frac{L-C_{0} \Omega}{\left[C_{0}+\frac{4}{3} \frac{k}{k_{\mathrm{s}}}\left(C_{0}-A_{0}\right)\right] \Omega} .
\end{aligned}
$$

We now substitute Eqs. (14) into (9) to get the variations of the inertia tensor due to the rotational deformation in terms of Andoyer variables. The result is:

$$
\begin{aligned}
& c_{11}=c_{22}=-\frac{2}{3} \frac{v}{C_{0}^{\prime}}\left(L-C_{0} \Omega\right) \\
& c_{33}=\frac{4}{3} \frac{v}{C_{0}^{\prime}}\left(L-C_{0} \Omega\right) \\
& D=\frac{v}{B_{0}} \sqrt{G^{2}-L^{2}} \cos l \\
& E=\frac{v}{A_{0}} \sqrt{G^{2}-L^{2}} \sin l \\
& F=0
\end{aligned}
$$

where

$v=\frac{k}{k_{\mathrm{s}}} \frac{1}{\Omega}\left(C_{0}-A_{0}\right) \quad$ and $\quad C_{0}^{\prime}=C_{0}+\frac{4}{3} \nu \Omega$.

Taking into account the relation between the variable $L$ and the angle $J: L=G \cos J$, we can express (15) as a function of $J$ as follows:

$$
\begin{aligned}
& c_{11}=c_{22}=-\frac{2}{3} \frac{v}{C_{0}^{\prime}}\left(G \cos J-C_{0} \Omega\right) \\
& c_{33}=\frac{4}{3} \frac{v}{C_{0}^{\prime}}\left(G \cos J-C_{0} \Omega\right) \\
& D=\frac{v}{B_{0}} G \sin J \cos l \\
& E=\frac{v}{A_{0}} G \sin J \sin l \\
& F=0 .
\end{aligned}
$$

To first order, we can consider the approximations $G \approx L \approx$ $C_{0} \Omega$ and $\cos J \approx 1$. Then the periodic additions to the moments and products of inertia with respect to the average principal axes become:

$$
\begin{aligned}
& c_{11}=c_{22}=c_{33} \approx 0 \\
& D=\beta C_{0} \sin J \cos l \\
& E=\alpha C_{0} \sin J \sin l \\
& F=0
\end{aligned}
$$

where the following notation was adopted:

$$
\alpha=\frac{k}{k_{\mathrm{S}}} \frac{C_{0}-A_{0}}{A_{0}} \quad \text { and } \quad \beta=\frac{k}{k_{\mathrm{s}}} \frac{C_{0}-A_{0}}{B_{0}} .
$$

Numerical values of these parameters (for the Earth and Mars) are presented in Tables 2 and 3. Note that for an Earth with rotational symmetry $\left(A_{0}=B_{0}\right): \alpha=\beta$.

Following Kubo (1991), $D$ and $E$ should be considered only as functions of time. So, we will denote $J$ and $l$ in Eq. (18) as $J^{\star}$ and $l^{\star}$. Numerically, they are equal to $J$ and $l$, respectively. The existence of non-elasticity causes a time lag $\delta$ between the rotational axis and the pole of the equatorial bulge due to the centrifugal force. Then, $l^{\star}$ should be equal to $l+\delta(\delta>0)$ and $D$ and $E$ should be written as:

$D=\beta C_{0} \sin J^{\star} \cos l^{\star}=\beta C_{0} \sin J^{\star} \cos (l+\delta)$

$E=\alpha C_{0} \sin J^{\star} \sin l^{\star}=\alpha C_{0} \sin J^{\star} \sin (l+\delta)$.

When these last expressions are substituted in the general expression of the Hamiltonian (7) one obtains, considering the approximation for the determinant $\Delta \approx A_{0} B_{0} C_{0}$ and neglecting terms of second and higher order:

$$
\begin{aligned}
\mathcal{K}= & \mathcal{K}^{R}-\frac{L}{A_{0} B_{0}} \sqrt{G^{2}-L^{2}} \sin J^{\star} \\
& \times\left[\alpha B_{0} \sin l^{\star} \sin l+\beta A_{0} \cos l^{\star} \cos l\right]
\end{aligned}
$$

where

$\mathcal{K}^{R}=\frac{1}{2}\left(\frac{\sin ^{2} l}{A_{0}}+\frac{\cos ^{2} l}{B_{0}}\right)\left(G^{2}-L^{2}\right)+\frac{1}{2} \frac{L^{2}}{C_{0}}$

is the Hamiltonian of the torque-free motion for a rigid body with a triaxial form (Souchay et al. 2003).

When $\alpha \approx \beta$, that is to say the body is quasi-triaxial, we can do the approximation $\alpha \approx \beta \approx \gamma=\frac{\alpha+\beta}{2}$ and introduce this last value, $\gamma$, into the Hamiltonian (21). This substitution leads to the following expression for the Hamiltonian:

$$
\begin{aligned}
\mathcal{K}= & \mathcal{K}^{R}-\frac{\gamma}{A_{0} B_{0}} L \sqrt{G^{2}-L^{2}} \sin J^{\star} \\
& \times\left[B_{0} \sin l^{\star} \sin l+A_{0} \cos l^{\star} \cos l\right] .
\end{aligned}
$$

Adopting the following notation:

$\bar{A}=\frac{A_{0}+B_{0}}{2} \quad$ and $\quad \varepsilon=\frac{B_{0}-A_{0}}{A_{0}+B_{0}}$

we can rewrite Eq. (23) as:

$$
\begin{aligned}
\mathcal{K}= & \mathcal{K}^{R}-\frac{\gamma}{\bar{A}} L \sqrt{G^{2}-L^{2}} \sin J^{\star} \\
& \times\left[(1+\varepsilon) \sin l^{\star} \sin l+(1-\varepsilon) \cos l^{\star} \cos l\right]
\end{aligned}
$$

and the Hamiltonian of the torque-free motion for a rigid body (22) as:

$\mathcal{K}^{R}=\frac{1}{2 \bar{A}}(1-\varepsilon \cos 2 l)\left(G^{2}-L^{2}\right)+\frac{1}{2} \frac{L^{2}}{C_{0}}$.

Notice also that in the expressions (25) and (26) $\varepsilon$ has a small value.

For the case where it is not possible to make the above approximation: $\alpha \approx \beta \approx \gamma=\frac{\alpha+\beta}{2}$, the Hamiltonian (21) can be rewritten as, in terms of $\bar{A}$ and $\varepsilon$ :

$$
\begin{aligned}
\mathcal{K}= & \mathcal{K}^{R}-\frac{L}{\bar{A}} \sqrt{G^{2}-L^{2}} \sin J^{\star} \\
& \times\left[\alpha(1+\varepsilon) \sin l^{\star} \sin l+\beta(1-\varepsilon) \cos l^{\star} \cos l\right] .
\end{aligned}
$$




\subsection{Variational equations}

Once we have obtained the Hamiltonian corresponding to the torque-free rotational motion of an elastic body in terms of Andoyer variables $(L, G, H, l, g, h)$, we can use the general Hamilton's equations of motion to establish the variational equations of the problem considered here (Kinoshita 1977):

$\frac{\mathrm{d}}{\mathrm{d} t}(L, G, H)=-\frac{\partial \mathcal{K}}{\partial(l, g, h)}$

$\frac{\mathrm{d}}{\mathrm{d} t}(l, g, h)=\frac{\partial \mathcal{K}}{\partial(L, G, H)}$

which express the time variations of Andoyer variables as functions of partial derivatives of the Hamiltonian.

Variational equations for $\alpha \approx \beta \approx \gamma=\frac{\alpha+\beta}{2}$ :

The substitution of the Hamiltonian (25) in the previous expressions gives us:

$\frac{\mathrm{d} L}{\mathrm{~d} t}=-\frac{\varepsilon}{\bar{A}} G^{2} \sin ^{2} J \sin 2 l$

$$
+\frac{\gamma}{\bar{A}} G L \sin J \sin J^{\star}[\sin \delta+\varepsilon \sin (2 l+\delta)]
$$

$\frac{\mathrm{d} G}{\mathrm{~d} t}=0$

$\frac{\mathrm{d} H}{\mathrm{~d} t}=0$

$\frac{\mathrm{d} l}{\mathrm{~d} t}=-\frac{L}{\bar{A}}\left(\frac{C_{0}-\bar{A}}{C_{0}}\right)$

$+\frac{\varepsilon}{\bar{A}} L \cos 2 l+\frac{\gamma}{\bar{A}} L[\cos \delta-\varepsilon \cos (2 l+\delta)]$

$\frac{\mathrm{d} g}{\mathrm{~d} t}=\frac{G}{\bar{A}}-\frac{\varepsilon}{\bar{A}} G \cos 2 l-\frac{\gamma}{\bar{A}} L[\cos \delta-\varepsilon \cos (2 l+\delta)]$

$\frac{\mathrm{d} h}{\mathrm{~d} t}=0$

and taking into account that $\frac{\mathrm{d} J}{\mathrm{~d} t}=-\frac{1}{G \sin J} \frac{\mathrm{d} L}{\mathrm{~d} t}$, the variational equation for the angle $J$ is given by:

$\frac{\mathrm{d} J}{\mathrm{~d} t}=\frac{\varepsilon}{\bar{A}} G \sin J \sin 2 l-\frac{\gamma}{\bar{A}} L \sin J^{\star}[\sin \delta+\varepsilon \sin (2 l+\delta)]$.

For later use it is convenient to write the time variations of Andoyer variables $(z)$ in the form:

$\frac{\mathrm{d} z}{\mathrm{~d} t}=\left.\frac{\mathrm{d} z}{\mathrm{~d} t}\right|_{\mathrm{r}}+\left.\frac{\mathrm{d} z}{\mathrm{~d} t}\right|_{\mathrm{d}}$

where $\left.\frac{\mathrm{d} z}{\mathrm{~d} t}\right|_{\mathrm{r}}$ is the corresponding part of a rigid body and $\left.\frac{\mathrm{d} z}{\mathrm{~d} t}\right|_{\mathrm{d}}$ is the new contribution due to the deformation. The later forms (29) and (30) show very well that the terms without the factor $\gamma$ correspond to the expressions for the torque-free motion of a rigid body (Souchay et al. 2003). Thus, the pure contribution due to the deformation is given by:

$\left.\frac{\mathrm{d} L}{\mathrm{~d} t}\right|_{\mathrm{d}}=\frac{\gamma}{\bar{A}} G L \sin J \sin J^{\star}[\sin \delta+\varepsilon \sin (2 l+\delta)]$

$\left.\frac{\mathrm{d} G}{\mathrm{~d} t}\right|_{\mathrm{d}}=0$

$\left.\frac{\mathrm{d} H}{\mathrm{~d} t}\right|_{\mathrm{d}}=0$

$$
\begin{aligned}
& \left.\frac{\mathrm{d} l}{\mathrm{~d} t}\right|_{\mathrm{d}}=\frac{\gamma}{\bar{A}} L[\cos \delta-\varepsilon \cos (2 l+\delta)] \\
& \left.\frac{\mathrm{d} g}{\mathrm{~d} t}\right|_{\mathrm{d}}=-\frac{\gamma}{\bar{A}} L[\cos \delta-\varepsilon \cos (2 l+\delta)] \\
& \left.\frac{\mathrm{d} h}{\mathrm{~d} t}\right|_{\mathrm{d}}=0
\end{aligned}
$$

and the corresponding pure contribution due to the deformation for the angle $J$ is given by:

$\left.\frac{\mathrm{d} J}{\mathrm{~d} t}\right|_{\mathrm{d}}=-\frac{\gamma}{\bar{A}} L \sin J^{\star}[\sin \delta+\varepsilon \sin (2 l+\delta)]$

\section{Variational equations. General case:}

The substitution of the Hamiltonian (27) into the general variational Eqs. (28) gives us the following expressions for the temporal variations of Andoyer variables and the angle $J$ :

$$
\begin{aligned}
\frac{\mathrm{d} L}{\mathrm{~d} t}= & -\frac{\varepsilon}{\bar{A}} G^{2} \sin ^{2} J \sin 2 l \\
& +\frac{G L}{\bar{A}} \sin J \sin J^{\star}[\rho \sin \delta+\tau \sin (2 l+\delta)] \\
\frac{\mathrm{d} G}{\mathrm{~d} t}= & 0 \\
\frac{\mathrm{d} H}{\mathrm{~d} t}= & 0 \\
\frac{\mathrm{d} l}{\mathrm{~d} t}= & -\frac{L}{\bar{A}}\left(\frac{C_{0}-\bar{A}}{C_{0}}\right)+\frac{\varepsilon}{\bar{A}} L \cos 2 l \\
& +\frac{L}{\bar{A}}[\rho \cos \delta-\tau \cos (2 l+\delta)] \\
\frac{\mathrm{d} g}{\mathrm{~d} t}= & \frac{G}{\bar{A}}-\frac{\varepsilon}{\bar{A}} G \cos 2 l \\
& -\frac{L}{\bar{A}}[\rho \cos \delta-\tau \cos (2 l+\delta)] \\
\frac{\mathrm{d} h}{\mathrm{~d} t}= & 0 \\
\frac{\mathrm{d} J}{\mathrm{~d} t}= & \frac{\varepsilon}{\bar{A}} G \sin J \sin 2 l \\
& -\frac{L}{\bar{A}} \sin J^{\star}[\rho \sin \delta+\tau \sin (2 l+\delta)] \\
& \\
&
\end{aligned}
$$

where

$\rho=\frac{1}{2}[\alpha(1+\varepsilon)+\beta(1-\varepsilon)]$ and $\tau=\frac{1}{2}[\alpha(1+\varepsilon)-\beta(1-\varepsilon)]$.

Notice that in these equations we have introduced two new parameters: $\rho$ and $\tau$, which are related to the elastic parameters $\alpha$ and $\beta$ and the triaxial parameter $\varepsilon$, therefore characterize the shape-configuration and elastic properties of the body. In the case of rotational symmetry $(A=B)$, we can remark that $\alpha=\beta$, then: $\rho=\alpha$ and $\tau=0$, which is Kubo's approximation. According to Eq. (19), we have $\frac{\alpha}{\beta}=\frac{B_{0}}{A_{0}}$. Then, in the treatment of the torque-free polar motion of a body with a marked triaxial shape, $\alpha$ and $\beta$ should be significantly different, so that a consideration of the terms depending of the parameter $\tau$ is necessary. 
The solution of this system of equations and its corresponding applications to the Earth's and Mars' free polar motion will be presented in the next sections.

\subsection{Comparison of the Hamitonian solution with the linearized Liouville equations}

The goal of this section is the derivation of the variational equations for $l$ and $J$, as formulated in the previous section, by means of linearized Liouville equations, to demonstrate the equivalence between the two different formalisms used in the literature. A different comparison between these two formulations for a purely elastic Earth model can also be seen in Sevilla \& Romero (1985). Nevertheless, we derive here in detail the equation corresponding to the angle $J$ since its variation has not been deeply studied till now. A simple presentation of Liouville equations is offered first.

For Tisserand axes, the Liouville equation is the fundamental equation for the mathematical description of free polar motion for a non-rigid body. It can be written as follows (Munk \& MacDonald 1975, p.10):

$\frac{\partial}{\partial t}(C \vec{\omega})+\vec{\omega} \times(C \vec{\omega})=\overrightarrow{0}$.

Here $\frac{\partial}{\partial t}$ denotes the time derivative in the body-fixed system (which rotates with the body) and the cross $(\times)$ denotes the vector product of two vectors.

We will now linearize the above equation. Taking into account the expressions (2)-(4), (10)-(12) and that $D, E, F$ and $\overrightarrow{\delta \omega}$ are considered small quantities, and only retaining linear terms, the Liouville equations become:

$$
\begin{aligned}
& A_{0} \dot{m_{1}}+\left(C_{0}-B_{0}\right) \Omega m_{2}+\dot{E}-D \Omega=0 \\
& B_{0} \dot{m_{2}}-\left(C_{0}-A_{0}\right) \Omega m_{1}+\dot{D}+E \Omega=0 \\
& C_{0} \dot{m_{3}}+\dot{C}=0
\end{aligned}
$$

the dot denoting a time derivative $\frac{\partial}{\partial t}$.

The substitution of the derivatives for $E, D$ and $c_{33}$ yields:

$$
\begin{aligned}
& \dot{m_{1}}+\bar{\alpha} m_{2}=0 \\
& \dot{m_{2}}-\bar{\beta} m_{1}=0 \\
& \dot{m_{3}}=0
\end{aligned}
$$

with

$\bar{\alpha}=\frac{\left(C_{0}-B_{0}-v \Omega\right) \Omega}{A_{0}+\nu \Omega}$ and $\bar{\beta}=\frac{\left(C_{0}-A_{0}-\nu \Omega\right) \Omega}{B_{0}+\nu \Omega}$.

In terms of the principal moments of inertia and Love numbers:

$$
\begin{aligned}
& \bar{\alpha}=\frac{\left(\frac{k}{k_{\mathrm{s}}} A_{0}-B_{0}+\left(1-\frac{k}{k_{\mathrm{s}}}\right) C_{0}\right) \Omega}{\left(1-\frac{k}{k_{\mathrm{s}}}\right) A_{0}+\frac{k}{k_{\mathrm{s}}} C_{0}} \\
& \bar{\beta}=\frac{\left(1-\frac{k}{k_{\mathrm{s}}}\right)\left(C_{0}-A_{0}\right) \Omega}{-\frac{k}{k_{\mathrm{s}}} A_{0}+B_{0}+\frac{k}{k_{\mathrm{s}}} C_{0}} .
\end{aligned}
$$

Note. For rotational symmetry $\left(A_{0}=B_{0}\right)$ :

$$
\bar{\alpha}=\bar{\beta}=\sigma_{\mathrm{C}}=\sigma_{\mathrm{E}} \frac{1-\frac{k}{k_{\mathrm{s}}}}{1+\frac{k}{k_{\mathrm{s}}} \frac{\sigma_{\mathrm{E}}}{\Omega}} \quad \text { Chandler frequency }
$$

$\left(\sigma_{\mathrm{E}}\right.$ : Euler frequency).
The solution of the first two equations of Eq. (37) gives us the components $m_{1}$ and $m_{2}$ that characterize a deviation of the Earth's rotation axis from the $z$-axis for an arbitrary nonrigid body, that is to say, the polar motion. Similarly, the third equation can be solved for $m_{3}$ to get variations of the speed of rotation, or variations of the length of day.

Inserting the relations (14) and its derivatives into the first two Liouville Eqs. (37) and also taking into account that $L=$ $G \cos J$ and considering the case when $\alpha \approx \beta \approx \gamma=\frac{\alpha+\beta}{2}$, we get:

$$
\begin{aligned}
\cos J \sin l \frac{\mathrm{d} J}{\mathrm{~d} t} & +\sin J \cos l \frac{\mathrm{d} l}{\mathrm{~d} t}+\frac{C_{0}-B_{0}}{B_{0}} \Omega \sin J \cos l \\
& -\gamma \Omega \sin J^{\star} \cos (l+\delta)=0
\end{aligned}
$$

$$
\begin{gathered}
\cos J \cos l \frac{\mathrm{d} J}{\mathrm{~d} t}-\sin J \sin l \frac{\mathrm{d} l}{\mathrm{~d} t}-\frac{C_{0}-A_{0}}{A_{0}} \Omega \sin J \sin l \\
+\gamma \Omega \sin J^{\star} \sin (l+\delta)=0 .
\end{gathered}
$$

By introducing the relation:

$\frac{C_{0}-B_{0}}{B_{0}}-\frac{C_{0}-A_{0}}{A_{0}}=-2 \frac{\varepsilon C_{0}}{\bar{A}}$

and multiplying Eq. (40) by $\sin l$ and Eq. (41) by $\cos l$, and then adding the two equations, we get the result:

$\frac{\mathrm{d} J}{\mathrm{~d} t}=\frac{\varepsilon}{\bar{A}} G \sin J \sin 2 l-\frac{\gamma}{\bar{A}} L \sin J^{\star} \sin \delta$.

In the same way, by multiplying Eq. (40) by $\cos l$ and Eq. (41) by $\sin l$, and after combination, we find:

$\frac{\mathrm{d} l}{\mathrm{~d} t}=-\frac{L}{\bar{A}}\left(\frac{C_{0}-\bar{A}}{C_{0}}\right)+\frac{\varepsilon}{\bar{A}} L \cos 2 l+\frac{\gamma}{\bar{A}} L \cos \delta$.

Notice that the two equations above give the same expressions as those for $\frac{\mathrm{d} l}{\mathrm{~d} t}$ and $\frac{\mathrm{d} J}{\mathrm{~d} t}$ in Eqs. (29) and (30). Thus, the equivalence between the two formulations, that is to say the Hamiltonian formulation and that obtained from Liouville equations, has been demonstrated.

\subsection{Transformation between the axis of angular momentum and the axis of rotation}

All the analytical expressions obtained in the preceding sections concerned the axis of angular momentum of the celestial body. Indeed, the polar motion which could be really observed is that of the axis of rotation of the body. Therefore, it is necessary to recapitulate and to give with precision how these two motions are related one to the other. Let us represent the motion of the axis of rotation of the body with respect to its axis of figure in polar coordinates $(\chi, \theta)$, where $\chi$ is the radius of the motion and $\theta$ the polar angle. They satisfy the following equations, from Eq. (14):

$$
\begin{aligned}
& m_{1}=\chi \sin \theta=\frac{1}{A_{0} \Omega} G \sin l \sin J \\
& m_{2}=\chi \cos \theta=\frac{1}{B_{0} \Omega} G \cos l \sin J .
\end{aligned}
$$


Thus, for very small $J(J \ll 1)$ and with the approximation $G \approx C_{0} \omega_{3} \approx C_{0} \Omega$, we get:

$\chi \sin \theta \approx \frac{C_{0}}{A_{0}} J \sin l$

$\chi \cos \theta \approx \frac{C_{0}}{B_{0}} J \cos l$

which gives the direct relationships:

$\tan l=\frac{A_{0}}{B_{0}} \tan \theta$

and, after transformation:

$l \approx \theta-\varepsilon \sin 2 \theta$.

Moreover, by combining the two equations of Eq. (44), we get to the first order the relationships between $J$ and $\chi$ :

$J \approx \frac{A_{0}}{C_{0}} \chi(1+\varepsilon \cos 2 \theta)$.

\subsection{Solution of the free polar motion equations with $\tau \ll 1$}

Neglecting second order terms, we have solved the variational Eqs. (33) for the variables $l$ and $J$. In the solution of these equations, we have considered that $\tau$ is negligible (see Tables 3 and 4, for the case of the Earth and Mars) and that $J$ has a very small amplitude so that we can write: $\sin J \approx \sin J^{\star} \approx J$. Then, on performing the integrations with respect to the time $t$, we have found the following explicit form of the solutions:

$l=-l_{1} t+l_{2} \sin \left(2 l_{1} t\right)$

$J=J_{0} \times \exp \left\{-J_{1} t-J_{2} \cos \left(2 l_{1} t\right)\right\}$

where the coefficients $l_{i}$ and $J_{i}$ are:

$l_{1}=\frac{C_{0} \Omega}{\bar{A}}\left[\frac{C_{0}-\bar{A}}{C_{0}}-\rho \cos \delta\right]$

$l_{2}=\frac{C_{0} \Omega \varepsilon}{2 \bar{A} l_{1}}$

$J_{0}=0.5 \times \exp \left\{\frac{C_{0} \Omega \varepsilon}{2 \bar{A} l_{1}}\right\}$

$J_{1}=\frac{C_{0} \Omega \rho}{\bar{A}} \sin \delta$

$J_{2}=\frac{C_{0} \Omega \varepsilon}{2 \bar{A} l_{1}}$.

Equations (49) and (50), together with the set of integration constants (51) give the features of the polar motion $(l, J)$ as deduced from our Hamiltonian equations and clearly show the effect of elasticity $(\rho)$, time lag $(\delta)$ and triaxiality $(\varepsilon)$.

From Eq. (49), we can observe that the angle $l$ not only undergoes the retrograde linear variation with frequency $-l_{1}$, corresponding to the Chandler motion, but also a superposed oscillation with frequency twice that of the Chandler motion and an amplitude which depends directly on the triaxial coefficient $\varepsilon$. The amplitude of the angle $J$ from Eq. (50) can be re-written, to first order, as:

$J=J_{0} \exp \left\{-J_{1} t\right\} \times\left(1-J_{2} \cos \left(2 l_{1} t\right)\right)+O\left(J^{2}\right)$.
The negative exponential coefficient $-J_{1}$ characterizes the damping of the polar motion, which depends directly on the phase lag $\delta$. Therefore, the variations of $J$ are characterized by a main component, decreasing with respect to the time, and an oscillation with amplitude $J_{0} J_{2} \exp \left\{-J_{1} t\right\}$ decreasing in the same proportion as this main component, and a frequency twice the frequency $l_{1}$ of the Chandler wobble. Notice that the coefficient $J_{2}$, and also the argument of the exponential function in $J_{0}$, can be written as:

$J_{0}=0^{\prime} 5 \times \exp \left\{\frac{C_{0} \varepsilon}{2} \frac{1}{C_{0}(1-\rho \cos \delta)-\bar{A}}\right\}$

$J_{2}=\frac{C_{0} \varepsilon}{2} \frac{1}{C_{0}(1-\rho \cos \delta)-\bar{A}}$

which depend on both the set of parameters $(\rho, \delta, \varepsilon)$ and the Chandler frequency. In the following a detailed practical application is made to the Earth and Mars.

\section{Application to the Earth}

Our purpose in this section is to show precisely what are the various influences acting on the true polar motion of the Earth and which are clearly represented by each of the components in the right-hand side of Eqs. (33) giving the expressions of $\mathrm{d} l / \mathrm{d} t$ and $\mathrm{d} J / \mathrm{d} t$. These are the influence of the triaxiality of the Earth, through the parameter $\varepsilon$, of the Earth's elasticity, through $\rho$, and of the time lag $\delta$ due to the inelastic response of the planet. For that we use actual polar motion data of the Earth, and as a first step we explain how the angles $J$ and $l$ which are fundamental in our equations, are determined starting from their counterparts for the axis of rotation, respectively $\chi$ and $\theta$, as determined from up-to-date observational data.

\subsection{Use of real polar motion data: The problem of determination of the origin}

In the equations of the preceding section the motion of the angular momentum axis is given by the two polar coordinates $l$ and $J$, with respect to figure axis. As we have shown in Sect. 3.4, the rotation axis is very close to the angular momentum axis, so that we make an a priori assumption that there is no angular difference between the two axes, and we will assume that $J$ and $l$ in our equations also characterize the motion of the pole of rotation. In the following we justify this approximation by calculating the quantities $l-\theta$ and $J-\chi$ as given by Eqs. (47) and (48).

A preliminary step consists in determining the real values of $\chi$ and $\theta$, which represent the polar coordinates of the axis of rotation of the Earth with respect to the axis of figure. For that we use the data of the IERS series C-04 in Cartesian coordinates $(x, y)$ of the pole of rotation with respect to an arbitrary point $O_{\mathrm{c}}$ adopted conventionally and internationally (IERS Annual Report, 2003). Notice that we restrict our study to the period ranging from 1971, Feb. 18th to 2004, Feb. 19th. It is obvious that the conventional point $O_{\mathrm{c}}$ is not appropriate for defining the $(\chi, \theta)$ data set, for $O_{\mathrm{c}}$ designates a point significantly different from the point where the axis of figure pierces the Earth's surface. 
Table 2. The choice of origin $O$ and its influence on the period of the Chandler wobble.

\begin{tabular}{|c|c|c|c|c|}
\hline $\begin{array}{l}\Delta x \\
\left({ }^{\prime \prime}\right)\end{array}$ & $\begin{array}{l}\Delta y \\
\left({ }^{\prime \prime}\right)\end{array}$ & $\begin{array}{l}\text { Rate } \\
(\mathrm{rd} / \mathrm{d})\end{array}$ & $\begin{array}{c}T_{\text {Chandler }} \\
\text { (d) }\end{array}$ & $\begin{array}{c}\text { Relative } \\
\text { uncertainty }\end{array}$ \\
\hline 0.05 & 0.27 & $-0.01450623 \times 10^{-6}$ & 433.138 & $9.63 \times 10^{-5}$ \\
\hline 0.07 & 0.27 & $-0.01479483 \times 10^{-6}$ & 433.176 & $1.01 \times 10^{-4}$ \\
\hline 0.09 & 0.27 & $-0.01450440 \times 10^{-6}$ & 433.191 & $1.12 \times 10^{-4}$ \\
\hline 0.05 & 0.29 & $-0.01450667 \times 10^{-6}$ & 433.126 & $9.42 \times 10^{-5}$ \\
\hline 0.07 & 0.29 & $-0.01450483 \times 10^{-6}$ & 433.179 & $1.00 \times 10^{-4}$ \\
\hline 0.09 & 0.29 & $-0.01450279 \times 10^{-6}$ & 433.242 & $1.13 \times 10^{-4}$ \\
\hline
\end{tabular}

For our present study, we will assume that it is represented by an origin $O$ (intersection of the figure axis with the Earth's crust) so that the real path of the rotation axis should satisfy symmetry around $O$. Therefore, the first step determines a suitable offset $O_{\mathrm{c}} O$ from $O_{\mathrm{c}}$ such that the polar angle $\theta$ has a regular behavior, as close as possible linear.

To do that, we tested a set of different origins $O$ corresponding to $\Delta x=\left\{0.05,0 .^{\prime} 07,0 .^{\prime} 09\right\}$ and $\Delta y=\left\{0 . .27,0 .{ }^{\prime} 29\right\}$ which seem at first sight to be very close to the best estimation. We then made a linear fit of $\theta$ and we calculated the slope from which we deduce the value of the period of the Chandler wobble, $T_{\text {Chandler }}$. Our results are shown in Table 2 . The relative uncertainty is equal to the uncertainty of the slope. The period of the Chandler wobble determined by the linear regression procedure outlined above varies between $433.126 \mathrm{~d}$ and $433.242 \mathrm{~d}$.

It can be shown that a significant drift of $O$ from the best location of the figure axis will result both in more significant variations of the amplitude of $\chi$ and $\theta$ (once the linear component for this last angle has been removed). Moreover, the signal for these two parameters should then be characterized by the presence, as an artificial effect, of a sinusoidal oscillation with a period equal to that of the polar motion (Chandler term). The larger this oscillation, the larger the departure of $O$ from its optimal choice. To illustrate this we show respectively in Figs. 1a, for $\chi$, and $1 \mathrm{~b}$, for $\theta$, the two curves corresponding to a different choice of the origin $O$. In the two figures the bold curve corresponds to $O_{\mathrm{c}} O=\left(0 .{ }^{\prime} 07,0.29\right)$, whereas the thin curve corresponds to $O_{\mathrm{c}} O=\left(0 .{ }^{\prime} 05,0 .{ }^{\prime} 27\right)$. The time $t$ is measured in days from the original date 1971, Feb. 20th, so that the 3000-day time interval ranges between 1982, Jan. 30th and 1990, Apr. 19th. We can note in the two figures that in the second case $\left(O_{\mathrm{c}} O=(0\right.$ ".07, 0 ".29)), an oscillation with period close to $T_{\text {Chandler }}$ appears clearly, as an artificial effect explained above, and due to an inappropriate choice of $O$, whereas this oscillation is not present in the first case. Then we can admit that the choice of the origin $O_{\mathrm{s}}$ such that the offset $O_{\mathrm{c}} O_{\mathrm{s}}=\left(0 \prime^{\prime} 05,0{ }^{\prime} 29\right)$ is a suitable one. In the following we will adopt this value. Notice that the time span considered (4000 d $<t<7000 \mathrm{~d}$ ) has been selected such that the signal does not present an artificial oscillation due to an inappropriate choice of $O$, as explained before. We also observed that the secular motion of the pole (Höpfner 2003) outside the time span above noticeably increases this oscillation.

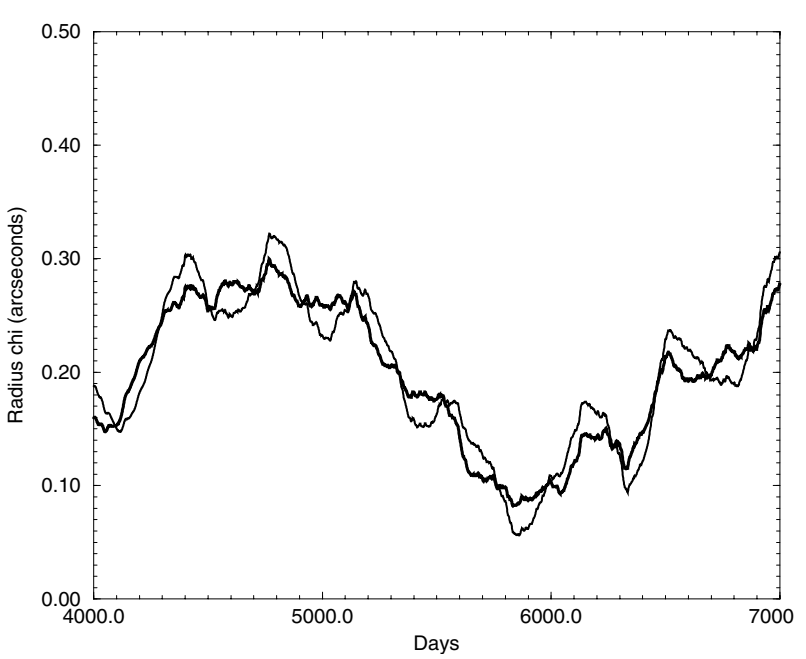

(a)

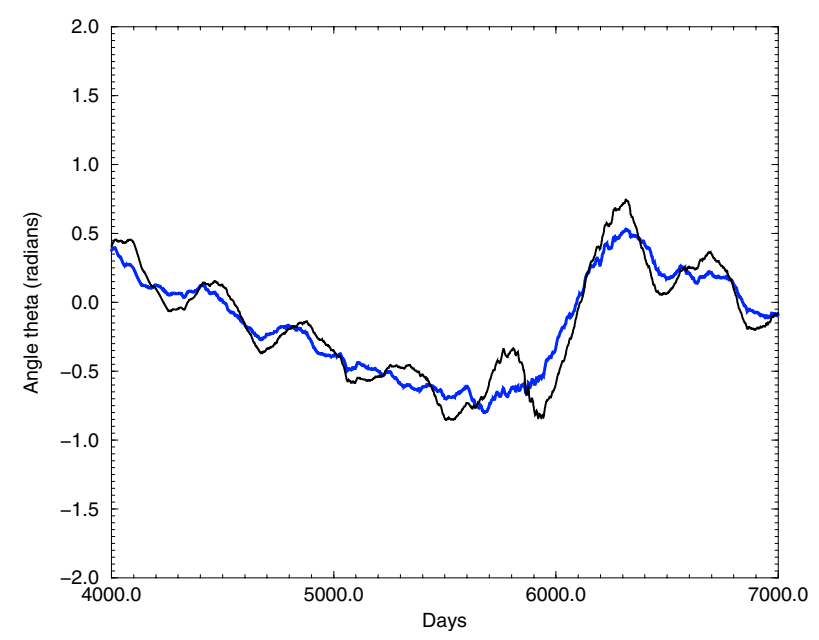

(b)

Fig. 1. Variation of the radius $\chi$ and the angle $\theta$ of the polar motion of the rotation axis with respect to the origin $O$ such that $O_{\mathrm{c}} O=$ $\left(0 .{ }^{\prime} 05 ; 0.27\right)$ (thin curve) and $O_{\mathrm{c}} O=\left(0.0^{\prime} 07 ; 0.29\right)$ (thick curve). The corresponding time span covers the range between 1982, Jan. 30th and 1990, Apr. 19th.

In Fig. 2 we show the classical $(x, y)$ two-dimensional representation of the polar motion centered on the selected origin $O_{\mathrm{s}}$, in the selected time span. We can remark that this curve has a circular shape and that the point $O$, represented by the black point at the center, looks like a suitable point of symmetry of the curve, which confirms its choice.

\subsection{Study of specific contributions to the polar motion}

We will now analyze the solutions $(d)$ and $(g)$ of Eq. (33) for the Earth's free polar motion $(J, l)$. Notice that we are not interested here in the question of how the Chandler wobble is maintained in the presence of dissipation, or in the exact nature of the excitation. This is the subject of abundant research at the present date. For a review of this topic, see e.g. Höpfner (2003).

We will focus in particular on showing how the time lag, $\delta$, plays an important role in the damping of this motion, and 


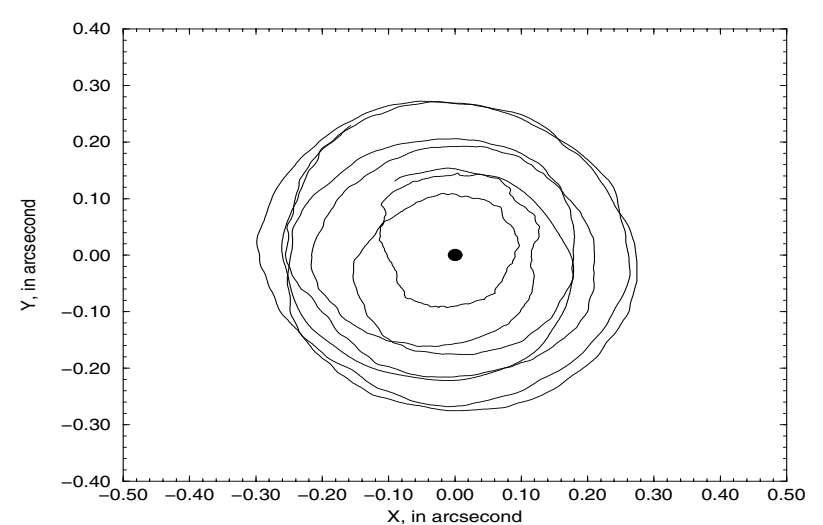

Fig. 2. $(x, y)$ coordinates of the pole of rotation of the Earth for the 3000-day time interval between 1982, Jan. 30th and 1990, Apr. 19th.

Table 3. The Earth parameters.

\begin{tabular}{llll}
\hline \hline Parameter & Value & Unit & Origin \\
\hline$A_{0}$ & $8.0101 \times 10^{37}$ & $\mathrm{~kg} \mathrm{~m}^{2}$ & Groten (2000) \\
$B_{0}$ & $8.0103 \times 10^{37}$ & $\mathrm{~kg} \mathrm{~m}^{2}$ & Groten (2000) \\
$C_{0}$ & $8.0365 \times 10^{37}$ & $\mathrm{~kg} \mathrm{~m}^{2}$ & Groten (2000) \\
$k$ & 0.29 & & \\
$k_{\mathrm{s}}$ & 0.9383 & & Groten (2000) \\
$\Omega$ & $7292115 \times 10^{-11}$ & $\mathrm{rad} \mathrm{s}^{-1}$ & Groten (2000) \\
$\alpha$ & $1.01864 \times 10^{-3}$ & & This paper \\
$\beta$ & $1.01861 \times 10^{-3}$ & & This paper \\
$\gamma$ & $1.01862 \times 10^{-3}$ & & This paper \\
$\bar{A}$ & $8.0102 \times 10^{37}$ & $\mathrm{~kg} \mathrm{~m}^{2}$ & This paper \\
$\varepsilon$ & $1.24841 \times 10^{-5}$ & & This paper \\
$\rho$ & $1.01863 \times 10^{-3}$ & & This paper \\
$\tau$ & $2.77167 \times 10^{-8}$ & & This paper \\
\hline
\end{tabular}

the influence of the polar motion amplitude itself on the irregular nature of the damping. But we want also to make specific conclusions based on the results established in the preceding sections, and which can be applied to the Earth. One is the evaluation of the pole offset between the angular momentum axis and the rotation axis. Another is the determination of the periodic components of the rotation axis of half the Chandler period whose existence and amplitudes can be deduced directly from Eqs. $(d)$ and $(g)$ of Eq. (33). Table 3 presents the Earth parameters that we have used in our computations. The numerical values for $\alpha, \beta, \gamma, \bar{A}, \varepsilon, \rho$ and $\tau$ for the Earth were determined from the expressions (19), (24) and (34).

\section{Period of the Chandler wobble}

Our fitted value for the period of the Chandler wobble as determined in the preceding section was $T_{\text {Chandler }} \approx-433.1 \mathrm{~d}$ (in this subsection we use the sign "-" to show that the motion is retrograde). Various studies have shown that this period should range between 410 and 442 days (Höpfner 2004), and our value is intermediate between these two extremes.

As summarized by Höpfner (2004), the contribution of each component to the Chandler period has been estimated: the elasticity of the mantle brings +143.0 days, the fluidity of the core -50.5 days, the pole tide (effect due to the oceans) +29.8 days,

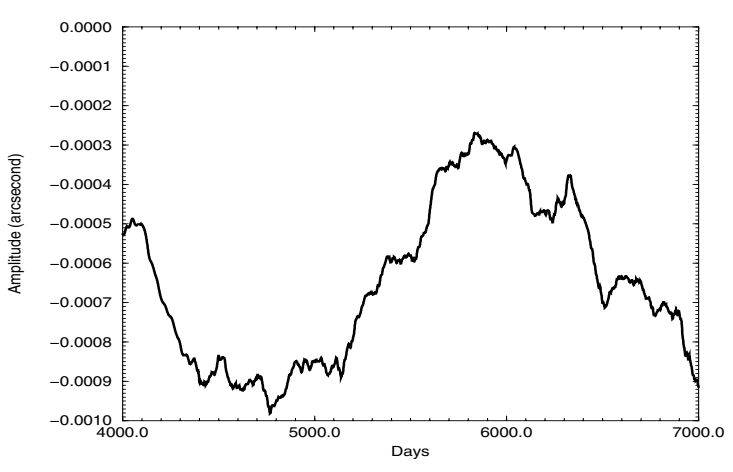

Fig. 3. Difference $J-\chi$ between the radius $J$ of the path described by the angular momentum axis and the radius $\chi$ of the corresponding rotation axis with respect to the origin $O_{\mathrm{s}}$ such that $O_{\mathrm{c}} O_{\mathrm{s}}=(0 . \prime 05 ; 0.29)$.

and the anelasticity of the mantle (dispersion) +8.5 days (Smith \& Dahlen 1981).

Notice that our Eq. (d) of Eq. (33) enables us to deduce directly from $i$ the analytical determination of the Chandler period taking into account only the elasticity of the mantle:

$i=\frac{C_{0} \omega}{\bar{A}} \times\left[-\frac{C_{0}-\bar{A}}{C_{0}}+\gamma \cos \delta\right]$

which gives $T_{\text {Chandler }}=2 \pi / \dot{l}=-442.2$ days. Moreover, this same value when considering the Earth as a rigid body, and corresponding to the Eulerian motion, is obtained by setting $\gamma=0$ in the equation above. We thus find: $T_{\text {Euler }}=-304.6$ days. Therefore the contribution of the Earth's elasticity coming from our calculations is 137.6 days which has to be compared with the 143 days above.

\section{Pole offset between the angular momentum axis and the rotation axis}

Figures 3 and 4 show respectively the differences of radius $J-\chi$ and of polar angle $l-\theta$ between the axis of angular momentum and the axis of rotation, as deduced from Eqs. (47) and (48), and where $\chi$ and $\theta$ are taken from the real data. The amplitude of $J$ is always smaller than the amplitude of $\chi$, with differences varying between -0.25 and -1.0 milliarcsec (Fig. 3). In comparison, the variations of $l-\theta$ (Fig. 4) are quite regular, with a quasi-sinusoidal behavior, with amplitude a little larger than $1^{\circ}$ and a period that is slightly variable and roughly

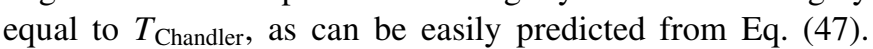
Notice that the separation between the two axes is not negligible when compared with the present observational precision of GPS and VLBI techniques (better than 0.'001), although it is small relative to the total amplitude of the polar motion.

\section{Determination of the phase lag $\delta$ responsible}

\section{for the damping}

The real value of $\delta$ has been subject to many investigations and is poorly known. According to the estimate of the quality factor $Q^{-1}$, which represents the dissipation inside the Earth, and the relationship between these damping parameters: $Q^{-1}=\tan \delta \approx \sin \delta$ (see e.g. Lambeck 1988), we can obtain the most probable value for the time lag. Some estimates of 


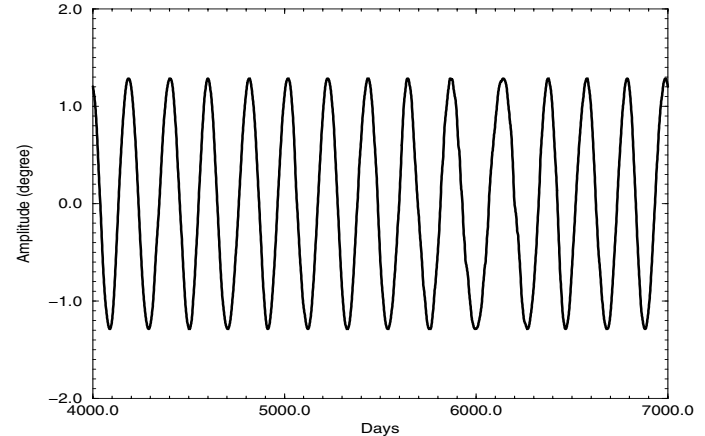

Fig. 4. Difference $l-\theta$ between the angle $l$ describing the path of the angular momentum axis and the angle $\theta$ of the corresponding path of the rotation axis with respect to the origin $O_{\mathrm{s}}$ such that $O_{\mathrm{c}} O_{\mathrm{s}}=$ $(0,05 ; 0.29)$.

the specific dissipation function have been computed by Okubo (1982), who showed that: $50 \leq Q \leq 100$, so that the range of $\delta$ should be roughly between 0.5 and 1.5 . More detailed aspects concerning the $Q$ factor can also be found in Widmer et al. (1991) and Mathews et al. (2002). The subject is an active one.

We have calculated the theoretical variations of $J$ and $l$ (or $\chi$ and $\theta$ ) given respectively by $(d)$ and $(g)$ of Eq. (33), according to $1^{\circ}$ incremental values of the phase shift $\delta$ from $\left(1^{\circ}\right.$ to $\left.6^{\circ}\right)$. The influence of $\delta$ on the damping of the angle $\chi$ is quite remarkable as can be seen in Fig. 5. We have fitted the variations of $\chi$ with a seventh order polynomial in order to enhance the trend and to compare with the long periodic damping effect. Notice that, between $t=5000 \mathrm{~d}$ and $t=6000 \mathrm{~d}$, there seems to be a period of damping accompanied by a significantly decreasing amplitude (from 0.3 to 0 !' 1 ) that appears to be in accordance with a value of $\delta \approx 6^{\circ}$, which is significantly larger than the value found by other authors. For instance, Williams et al. (1978) and Bois et al. (1996), starting from the acceleration of the Moon's motion due to tides, consider a time lag of $0.041 \mathrm{rd}$ and $0.054 \mathrm{rd}$, respectively, corresponding roughly to 2.4 and $3^{\circ}$. Notice also that the effect of damping is not linear with respect to time, for its derivative is directly proportional to the amplitude $\chi$ of the motion.

\section{Determination of the amplitude of periodic analytical contributions}

In Sect. 3.3 we have shown and defined with precision the existence of sinusoidal oscillations, with frequency $2 \dot{l}$, (or $2 \dot{\theta}$ ) both for $J$ and $l$ (or respectively for $\chi$ and $\theta$ ). These oscillations come from the first and third term containing $2 l$ of the right hand-side of Eq. $(d)$ in Eq. (33) and the two terms on the right hand-side of $(g)$ in Eq. (33). These equations give respectively the expressions for $\mathrm{d} J / \mathrm{d} t$ and $\mathrm{d} l / \mathrm{d} t$. We have integrated these equations using the real values of $J$ and $l$ for the Earth in the 3000-day time interval already chosen, and inserted them in the equations. The results are shown respectively in Figs. 6 and 7 for $\chi$ and $\theta$. As we think that it is of some interest to compare these contributions with the amplitudes of the differences $J-\chi$ and $l-\theta$ already shown in Figs. 3 and 4, these are presented again in dashed lines.

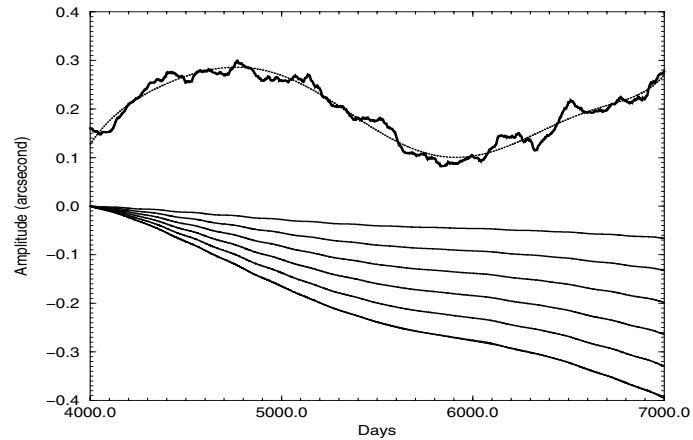

Fig. 5. The same curve as Fig. 1 representing the radius $\chi$ of the polar motion of the rotation axis, fitted by a 7th. order polynomial. The six curves at the bottom represent the damping effect as calculated by Eqs. (33), for different values of the phase lag $\delta$, from $1^{\circ}$ (upper curve) to $6^{\circ}$ (lower curve), with $1^{\circ}$ increment.

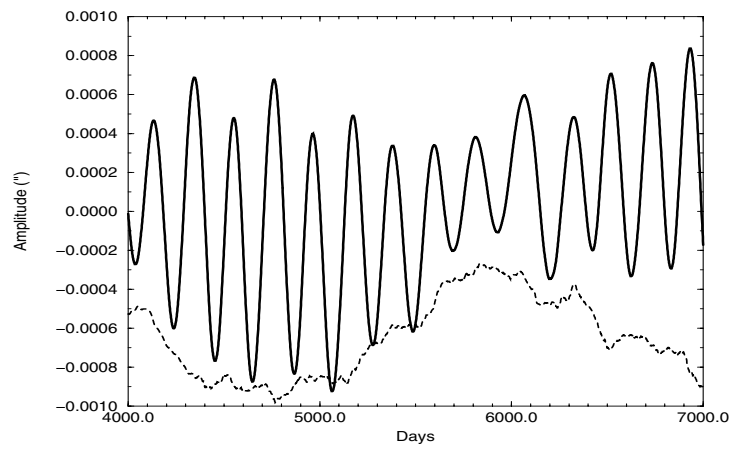

Fig. 6. Short-period oscillations, with period $T_{\text {Chandler }} / 2$, calculated from Eq. (33), characterizing the radius $\chi$ of the polar motion of the axis of rotation of the Earth, with respect to the origin $O_{\mathrm{s}}$. For comparison, the difference $J-\chi$ between the two axes (Fig. 3) is shown as a dashed line.

The curve for $J$ (Fig. 6) consists of large oscillations in amplitude of between 0.4 and 1.6 milliarcsec peak-to-peak, and for which the $2 i$ frequency appears clearly. Notice that the amplitudes of these oscillations are significantly larger than the amplitudes of the differences of radius between the two axes. Moreover, they are larger than the level of precision of the GPS and VLBI determinations of the polar motion $(x, y)$ (a few 0.1 mas). Thus they might a priori be detected, and it seems instructive to show in parallel the short periodic curve of the variations of $\chi$ obtained by subtracting the 7 th. order fitting polynomial from the global variation in Fig. 5. The results are shown in Fig. 7. The amplitude of these variations is larger by one order of magnitude, so that the detection of the effects discussed here might not be possible.

On the other hand, in Fig. 8 the amplitude of the $\chi$ oscillations with a frequency $2 \dot{l}$ (full line) is significantly smaller than that of the differences $J-\chi$ of the angle between the axis of figure and the axis of rotation (dashed line).

\section{Application to Mars}

As one of our main purposes in this paper was to set up in a straightforward and clear way the equations giving the polar motion $(J, l)$ of any given celestial body with irregular shape 


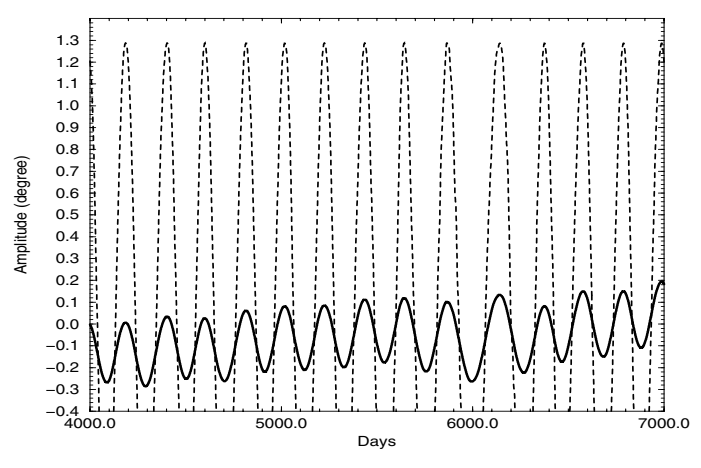

Fig. 7. Short-period oscillations, calculated from Eq. (33) with pe$\operatorname{riod} T_{\text {Chandler }} / 2$, characterizing the angle $\theta$ of the polar motion of the axis of rotation of the Earth with respect to the origin $O_{\mathrm{s}}$. For comparison, the difference $l-\theta$ between the two axes (Fig. 4) is shown in dashed line.

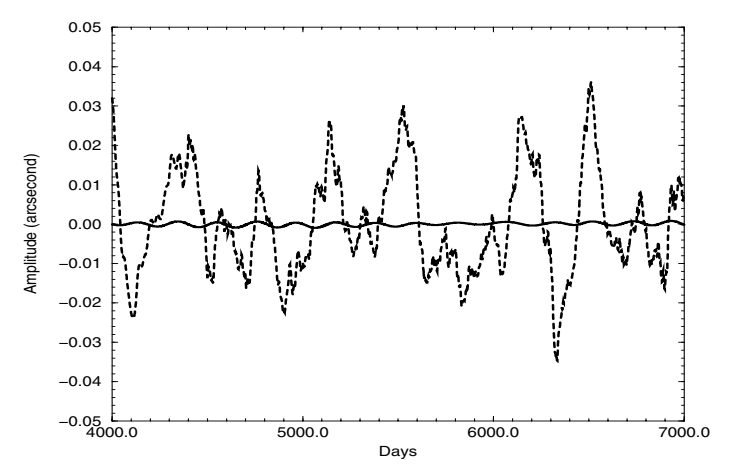

Fig. 8. Short-period oscillations of $\chi$ according to Eq. (33) already presented in Fig. 6 (thick line) in comparison with the short-period variations of $J-\chi$ (dashed line).

and elastic properties, according to the set of Eqs. (33), we show in this section how they can be applied advantageously in the case of Mars, whose physical characteristics, compiled in Table 4 from several works and recent modelings of the rotation of Mars such as Yoder \& Standish (1997), Folker et al. (1997), Bouquillon \& Souchay (1999) and Gauchez \& Souchay (2000), are rather well known. The numerical values for the elastic parameters and $\bar{A}$ and $\varepsilon$ for Mars were determined from the expressions (19), (24) and (34). Since accurate determinations of the damping parameter $\delta$ and the Love number of Mars are quite difficult to obtain, we have considered in this paper a wide range of values for $\delta_{\text {Mars }}$ (between 0.5 and $10^{\circ}$ ) and two extreme values for $k_{\text {Mars }}(0.15$ and 0.25$)$ to show the clear influence of both quantities on the free polar motion of Mars.

As we have no information on the excitation to which the polar motion of Mars should be subject, we have only considered the damping effect which leads to the exponentially decreasing tendency of the polar motion radius $J_{\text {Mars }}$ shown by the formula (50) depending on the three parameters $J_{0}$, $J_{1}$ and $J_{2}$. In Fig. 9 we have plotted the variations of $J_{\text {Mars }}$ (with an initial amplitude of 0.5 ) associated with different values of the time lag $\delta_{\text {Mars }}$ and Love number $k_{\text {Mars }}$. They clearly show the linear tendency of such solutions. Sine (or cosine) curves are superposed on these linear components, principally due to the effects (damping, triaxiality and elasticity) considered in the calculations. In particular, we note a large

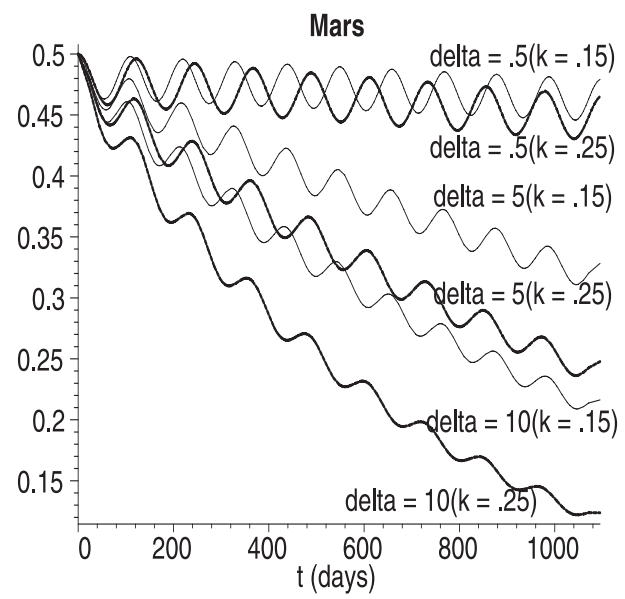

Fig. 9. Global solution of $J$ for Mars with changing $k$ and $\delta$. The units are in arcseconds.

dependence of the slope of the curve on the Love number adopted. Mathematically, this comes from the dependency of $J_{1}$ on the coefficient $\rho$ which is deduced from $k_{\text {Mars }}$ itself.

The periodic parts of the solutions for $J_{\text {Mars }}$ and $l_{\text {Mars }}$ are represented respectively in Fig. 10, for $\delta=5^{\circ}$ and $k=$ $(0.15,0.25)$. Notice that the curve for $k_{\text {Mars }}=0.15$ varies more quickly than that for $k=0.25$. This is again due to the presence of $l_{1}$ in the argument of the sine and cosine functions and also in the coefficient $J_{2}$, closely related to the damping. Consequently, when $k$ increases, $l_{1}$ diminishes slightly and $J_{2}$ gets larger. The corresponding period is lengthened. The damping is more significant for $k=0.25$ than that for $k=0.15$. Notice also that the amplitudes of these oscillations for $J_{\text {Mars }}$ and $l_{\text {Mars }}(t=0)$ are respectively $0 .{ }^{\prime} 8$ peak-to-peak and 2.3 , which is considerably larger than those corresponding to the Earth. Therefore, in the frame of future space missions, it is necessary to take into account such oscillations, in case excitation still prevails on the planet. Finally, in Fig. 11, we have plotted the $m_{i}$-components $(i=1,2)$ for different values of the Love number $k$ and for $\delta=5^{\circ}$

\section{Concluding remarks}

In this paper, we have adopted a Hamiltonian approach to the theory of the rotation of a triaxial and elastic celestial body to give the equations of the polar motion $(l, J)$. This follows the pioneer work of Kubo (1991), by taking into account not only elastic characteristics of the body but also the presence of a phase shift $\delta$ that represents a lag of the readjustment of masses inside the body, and also the influence of its triaxiality. In order to validate our analytical expressions, we have compared those for $J$ and $l$ with the ones obtained from the linearized Liouville equations, thus showing that they are equivalent to first order.

As the torque-free rotational motion of a celestial body has a direct dependence on its moments and products of inertia, the comparisons between their analytical solutions and present or future observational data can therefore provide useful material to understand the internal behavior of this body, as is already the case for the Earth. Some interesting connections may be found, for instance with slow changes in the figure of the Earth 

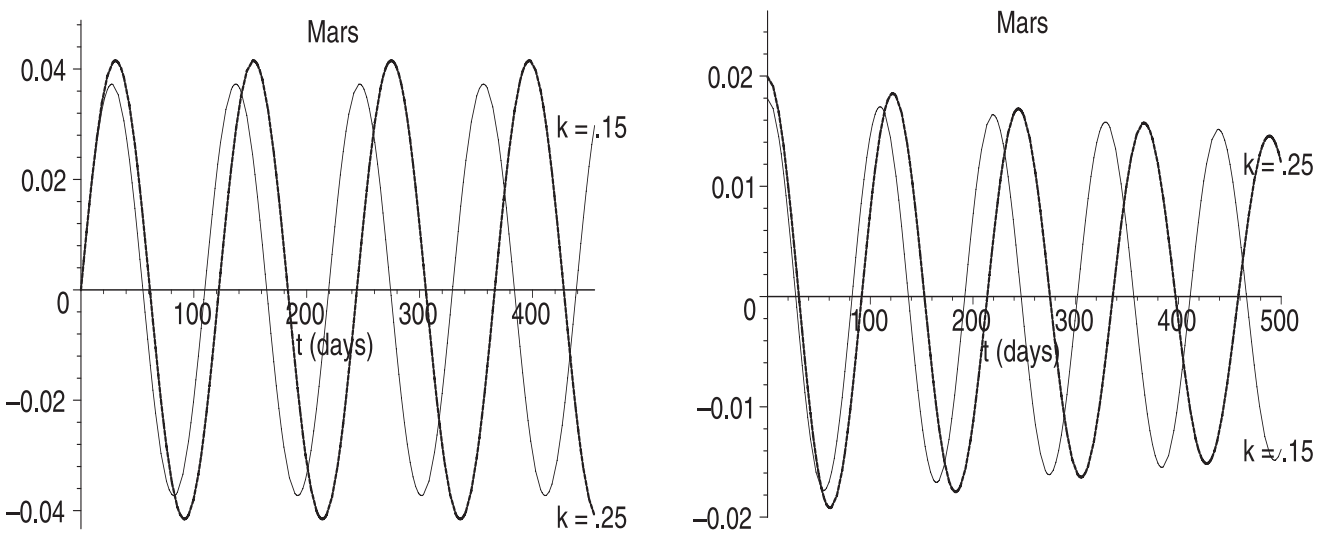

Fig. 10. Periodic part of $l$ and $J$ for $\delta=5^{\circ}$ and $k=(0.15,0.25)$. The units for $l$ are in radians and for $J$ in arcseconds.
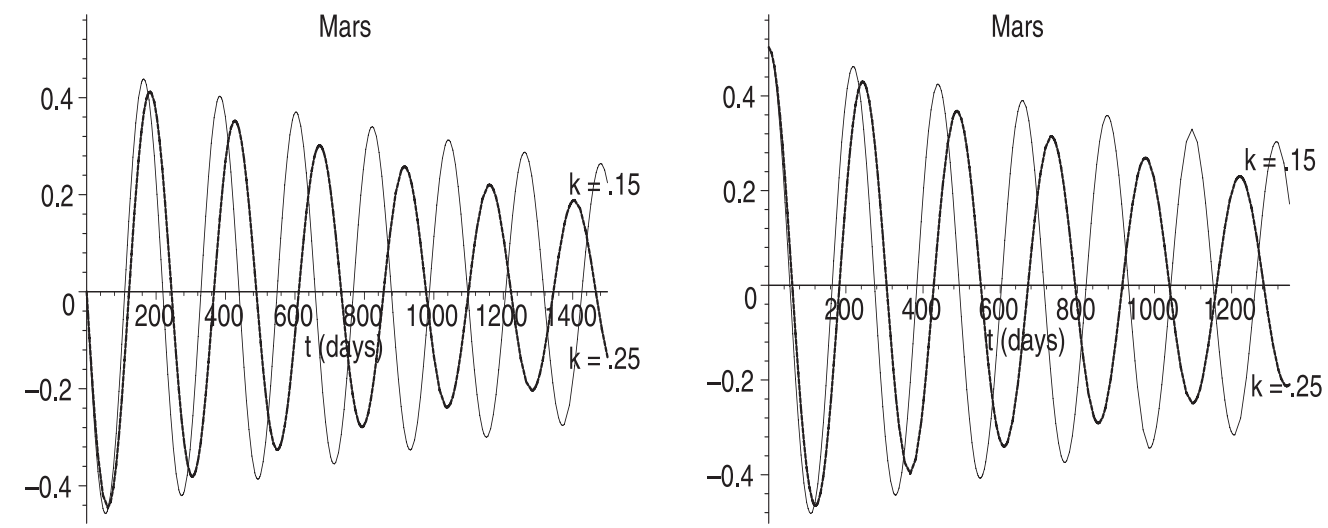

Fig. 11. $m_{1}$ and $m_{2}$-components for $\delta=5^{\circ}$. The units are in arcseconds.

Table 4. Parameters of Mars.

\begin{tabular}{llll}
\hline \hline Parameter & Value & Unit & Origin \\
\hline$\frac{C_{0}}{A_{0}}$ & 1.005741 & Bouquillon \& Souchay (1999) \\
$\frac{C_{0}}{B_{0}}$ & 1.005044 & \\
$B_{0}-A_{0}$ & 0.0006896 & Bouquillon \& Souchay (1999) \\
$C_{0}$ & & \\
$\frac{C_{0}}{M R^{2}}$ & 0.3662 & Bouquillon \& Souchay (1999) \\
& & \\
$k$ & $0.15-0.25$ & Folkner et al. (1997) \\
$k_{\mathrm{s}}$ & 1.203 & \\
$\Omega$ & $7.08822 \times 10^{-5}$ & rad s ${ }^{-1}$ & Folkner et al. (1997) \\
$\alpha(k=0.15)$ & $7.15836 \times 10^{-4}$ & This paper \\
$\beta(k=0.15)$ & $7.1534 \times 10^{-4}$ & This paper \\
$\gamma(k=0.15)$ & $7.15588 \times 10^{-4}$ & This paper \\
$\alpha(k=0.25)$ & $1.19306 \times 10^{-3}$ & This paper \\
$\beta(k=0.25)$ & $1.19223 \times 10^{-3}$ & This paper \\
$\gamma(k=0.25)$ & $1.192645 \times 10^{-3}$ & This paper \\
$\bar{A}$ & $2.68657 \times 10^{21}$ & $\mathrm{~kg} \mathrm{~m}{ }^{2}$ & This paper \\
$\varepsilon$ & $0.346625 \times 10^{-3}$ & This paper \\
$\rho(k=0.15)$ & $0.71559 \times 10^{-3}$ & This paper \\
$\tau(k=0.15)$ & $0.49604 \times 10^{-6}$ & This paper \\
$\rho(k=0.25)$ & $0.11926 \times 10^{-2}$ & This paper \\
$\tau(k=0.25)$ & $0.82840 \times 10^{-6}$ & This paper \\
\hline & &
\end{tabular}

and other celestial bodies, as well as in the relationships between the pole of figure and rotation.

We have applied our analytical calculations for the Earth to emphasize small but significant contributions to the polar motion. At first, as our two-dimensional parametrization of the angular momentum pole and of the rotation pole of the Earth with respect to a given origin is done though polar coordinates - respectively $(J, l)$ and $(\chi, \theta)$ - instead of the usual $(x, y)$ rectangular representation, we have studied in detail how the choice of the origin influences both the values of the coordinates and the determination of the Chandler period $T_{\text {Chandler. }}$. We have shown that for our selection of real data of polar motion between 1982, Jan. 30th and 1990, Apr. 19th, this motion is very regular around the point with coordinates $O_{\mathrm{c}} O_{\mathrm{s}}=\left(0{ }^{\prime} .05,0{ }^{\prime} 29\right)$ with respect to the conventional CIP pole, and that this point seems to be the most appropriate choice for the origin.

We also thought it was of some interest to evaluate the differences in amplitude $(J-\chi)$ and in angle $(l-\theta)$ between the axis of angular momentum and the axis of rotation. Moreover we have shown that in addition to the classical Chandlerian component of the polar motion, dampened by the influence of the phase lag $\delta$, an oscillation exists for both for $J$ and $l$, with frequency twice the frequency of the Chandler wobble itself and an amplitude directly proportional to the coefficient $\varepsilon$ characterizing the triaxiality.

One of our important results concerns the value of the phase lag $\delta$ responsible for the damping. For that we selected a 
time interval for which the motion of the pole clearly undergoes damping, and we could show that according to our simulations, a value of $\delta \approx 6^{\circ}$ seems in accordance with the negative slope caused by the decrease of the angle $\theta$; this value is significantly larger than that usually found in the literature.

Finally, we showed what should be the behavior of the polar motion for the planet Mars, and the dependence on parameters which are still poorly know, such as the Love number $k_{\text {Mars }}$ and the rotational phase lag $\delta_{\text {Mars }}$. We emphasize the fact that the oscillations with period $T_{\text {Chandler }} / 2$ are very large and of the same order as the amplitude of the polar motion itself, because of a more pronounced triaxial shape than for the Earth.

Acknowledgements. This research was carried out in the Department of "Systèmes de Référence Temps Espace" (SYRTE), Paris Observatory, under a combination of a financial support of Paris Observatory (2003) and Descartes Prize Allowance (2004) (M. Folgueira). Special thanks to Dr. D. McCarthy for several suggestions clarifying the presentation.

\section{References}

Bois, E., Boudin, F., \& Journet, A. 1996, A\&A, 314, 989

Bouquillon, S., \& Souchay, J. 1999, A\&A, 345, 282

Dehant, V., Arias, F., Bizouard, Ch., et al. 1999, Celest. Mech., 72(4), 245

Folkner, W. M., Yoder, C. F., Yuan, D. N., et al. 1997, Science, 278

Gauchez, D., \& Souchay, J. 2000, Earth, Moon and Planets, 84, 33

Getino, J., \& Ferrándiz, J. M. 1990, Celest. Mech., 49, 303

Getino, J., \& Ferrándiz, J. M. 1991, Celest. Mech., 51, 17

Groten, E. 2000, J. Geodesy, 74-1, 134

Höpfner, J. 2003, J. Geodesy, 77, 388

Höpfner, J. 2004, Surveys Geophys., 25, 1
Kinoshita, H. 1972a, Publ. Astron. Soc. Japan, 24, 409

Kinoshita, H. 1972b, Publ. Astron. Soc. Japan, 24, 423

Kinoshita, H. 1977, Celest. Mech., 15, 277

Kinoshita, H. 1992, Celest. Mech., 53, 365

Kubo, Y. 1991, Celest. Mech., 50, 165

Lambeck, K. 1980, The Earth's variable rotation (Cambridge University Press)

Lambeck, K. 1988, Geophysical Geodesy. The slow deformations of the Earth (Oxford: Clarendon Press)

Mathews, P. M., Herring, T. A., \& Buffett, B. A. 2002, J. Geophys. Res., 107, B4

McCarthy, D., \& Petit, G. 2003, IERS Conventions 2003, IERS Technical Notes 32

Moritz, H., \& Mueller, I. I. 1987, Earth rotation: Theory and Observation (New York: The Ungar Publishing Company)

Munk, W. H., \& MacDonald, G. J. F. 1975, The rotation of the Earth: A geophysical discussion (Cambridge University Press)

Nastula, J., \& Ponte, R. M. 1999, Geophys. J. Int., 139, 123

Okubo, S. 1982, J. Geophys. J. R. Astr. Soc., 71, 647

Sevilla, M. J., \& Romero, P. 1985, in Proc. of I Hotine-Marussi Symposium on Mathematical Geodesy, 703

Smith, M. L., \& Dahlen, F. A. 1981, Geophys. J. R. Astr. Soc., 64, 223

Souchay, J., Folgueira, M., \& Bouquillon, S. 2003, Earth, Moon and Planets, 93, 107

Stavinschi, M., \& Souchay, J. 1994, in Proc. of Journées, Systèmes de Référence spatio-temporels, 81

Van Hoolst, T., \& Dehant, V. 2002, Physics of the Earth and Planetary Interiors, 134, 17

Widmer, R., Masters, G., \& Gilbert, F. 1991, Geophys. J. Int., 104, 541

Williams, J. G., Sinclair, W. S., \& Yoder, C. F. 1978, Geophys. Res. Lett. 5, 11, 943

Yoder, C. F., \& Standish, E. M. 1997, J. Geophys. Res., 102(2), 4065 